\title{
A Coupling Framework for AspectJ
}

\author{
Marc Bartsch, Rachel Harrison \\ School of Systems Engineering, The University of Reading, \\ Reading, Berkshire, RG6 6AY, UK \\ \{m.bartsch, rachel.harrison\}@reading.ac.uk
}

\section{EXTENDED ABSTRACT}

Aspect-orientation is an emerging paradigm that is based on the separation of concerns principle. It offers the idea of a new modular unit that encapsulates crosscutting concerns which would otherwise be scattered across multiple modules. Aspect-oriented measurement is a research area that has gained an increasing amount of attention lately due to the definition of several suites of measures designed to support aspect-oriented key features. Unlike objectorientation, however, aspect-orientation lacks a sound foundation on which measures can be expressed in operationalisable ways making it difficult to analyse and compare existing aspect-oriented measures. We identify a need to create common ground on which the definition of aspect-oriented measures can be based.

Coupling in aspect-oriented systems lacks a rigorous investigation and given the fact that a high proportion of all specified aspect-oriented measures are for coupling, there is a need for a framework that focuses on this. In order to work towards such a framework, the unified framework for coupling measurements by Briand et al. (1999) has been used here as a suitable basis since one of the design goals of this framework has been an easy extensibility for coupling scenarios unknown in object-oriented languages. It offers six criteria that need to be considered when object-oriented measures are designed, analysed or compared. The six criteria are: type of connection, locus of impact, granularity, stability of server, direct or indirect connections and inheritance. We investigated and extended each criterion of this framework with regards to AspectJ (2005) which is the currently predominant implementation of an aspect-oriented language. In addition, we extended the framework by another criterion, describing counting rules based on built-in instantiation mechanisms for aspects.

A main focus of the extended framework is on the specification of the different kinds of coupling mechanisms that occur in AspectJ. We identified 5 groups of coupling mechanisms: join point based coupling, type pattern based coupling, identifier based coupling, reference based coupling and invocation based coupling. Join point coupling describes coupling that is caused by the reference of join points of an advice through the use of pointcut expression. Type pattern based coupling refers to coupling through the use of explicitly named classes or interfaces in pointcut expression. Identifier based coupling is a known coupling mechanism in object-oriented languages. In the context of AspectJ, identifiers of classes or aspects are used, for example, in attribute or method inter-type declarations, in declare parents, declare precedence or declare soft expressions, as return types or as parameters. Reference based coupling is also a known coupling mechanism. It occurs if one class or aspect references an attribute of another class. In an aspect, attributes can be referenced in methods, in method inter-type declarations, in advice or in pointcut expressions. The last group of coupling mechanisms is invocation based coupling, which occurs when a method, a method inter-type declaration, advice or a pointcut expression calls a method.

We applied the framework to define a new metric ACFC (Aspect Control Flow Changes) that has the measurement goal to capture the amount of control flow changes within a given class that are caused by aspects. This metrics is based on the hypothesis that control flow changes caused by aspects have an effect on the understandability of how a class relates to the rest of the system.

Our conclusion is that the coupling framework for AspectJ is a suitable means to express aspect-oriented coupling measures in a well-defined manner. The underlying coupling framework by Briand et al. has been found very flexible to allow its adaptation to aspect-orientation.

Future research will be focused on the application of this framework to investigate the well-definedness of existing aspect-oriented coupling measures. Our aim is the development of a set of theoretically and empirically validated aspect-oriented coupling measures. Other areas of research include the extension of object-oriented cohesion frameworks for the aspect-oriented paradigm.

\section{REFERENCES}

AspectJ, AspectJ Programming Guide (2005), http://eclipse.org/aspectj/doc/released/progguide/index.html

Briand, L.C., Daly, J.W. and Wüst, J.K. (1999) A Unified Framework for Coupling Measurement in Object-Oriented Systems. IEEE Transactions on Software Engineering, 25(1), 91-120. 